\title{
3D TENDON STRAIN ESTIMATION ON HIGH-FREQUENCY 3D ULTRASOUND IMAGES A SIMULATION AND PHANTOM STUDY
}

\author{
C. Carvalho ${ }^{\star}$ \\ S. Bogaerts \\ L. Scheys $s^{\dagger}$ \\ J. D’hooge \\ K. Peers \\ P. Suetens ${ }^{\star}$ \\ * KU Leuven, ESAT/PSI UZ Leuven, MIRC iMinds, Medical IT Dept, KU Leuven, and UZ Leuven, \\ ${ }^{\diamond}$ Dept. of Development and Regeneration, ${ }^{\dagger}$ Dept. of Orthopedics, ${ }^{\ddagger}$ Dept. of Cardiovascular Sciences
}

\begin{abstract}
Tendon strain is a topic of interest within the orthopaedics and sports medicine community. If accurately estimated, it can improve existing treatment and rehabilitation protocols and aid in detection of presymptomatic abnormalities. This paper presents a novel US-based strain estimation framework that integrates an affine image registration approach to quantify tendon strain with a high-resolution 3D US imaging system. Validation of this framework was performed on simulated and phantom data. An accuracy test of the acquisition system and the performance of 3D and 2D strain estimations were evaluated. Results show that attention should be paid to the acquisition protocol, best accuracy is obtained for simulation data and along the major deformation direction and 3D strain estimations seems to reduce out-of-plane effect. By using this technique, it is expected that clinicians expand knowledge on aetiology of tendinopathy and optimize the existing therapeutic programs. Furthermore this technique can be extrapolated to other tendons and ligaments that are vulnerable to overuse.
\end{abstract}

Index Terms - 3D tendon strain, high-frequency 3D US, image registration

\section{INTRODUCTION}

Tendinopathies are among the most common musculoskeletal injuries affecting recreational and elite athletes. Etiology is multifactorial, but mechanical overloading appears to be at the base of the problem [1]. The clinical diagnosis is usually confirmed using 2D ultrasound (US) imaging. This evaluation is based on tissue's hypo- or hyperechoicity, structures size, echoheterogeneity and arrangement and by the presence of shadows. Notwithstanding its undeniable clinical value, limitations can arise due to its qualitative nature.

Quantitative analysis of US images, on the other hand, allows a more objective analysis of the tissue's biomechanical parameters providing more clinically useful information. More specifically, local-intratendinous strain estimation would reveal the underlying behaviour of the tendon [2]. Difficulty in obtaining ground-truth for non-uniform strain distribution makes global strain estimation the first step towards the validation of strain estimation methods.

From an acquisition point-of-view, the combination of high-frequency US with 3D imaging presents several diagnostic and methodological advantages. 3D imaging reduces subjectivity of diagnosis and allows a better spatial and anatomical understanding of the scanned region. Moreover, high-frequency US transducers lead to texturally richer images with higher spatial resolution, allowing visualization up to the level of the tendon fascicles.

This paper presents a novel US-based strain estimation framework that integrates an affine image registration approach to quantify global deformation with a high-frequency 3D US imaging system. In addition we report the results of simulation- and phantom-based validation experiments.

\section{METHODS}

\subsection{High-frequency 3D US system}

US quantification is strongly dependent on the US images used as input. For this reason, a high-frequency 3D US system ( Vevo2100 FUJIFILM VisualSonics, Inc. , Toronto,CA) was chosen. To acquire 3D images, the transducer is mounted on a stepper motor (fixed to a mechanical arm) and a 2D image is capture at each step of the motor. At the end of the sweep, the collected 2D images are concatenated into a 3D US image in which the motor's step-size defines the elevation resolution. A transducer with a central frequency of $20 \mathrm{MHz}$ was used returning images with a spatial resolution of $0.02 \mathrm{~mm}$ along the axial direction, $0.09 \mathrm{~mm}$ along the lateral direction and $0.03 \mathrm{~mm}$ along the elevation direction. An example of an in-vivo 3D US image acquired using this system is presented in Fig.1.

\subsection{Strain estimation method}

The aim of image registration used in this work is to find a global transformation that maximizes spatial correspondences between deformed and undeformed images. Computer simulated data was used to tune the image registration parameters and sum of squared differences was the used metric, a quasi- 


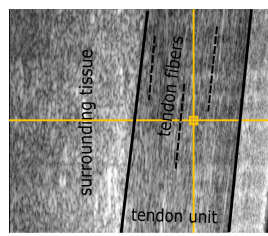

(a)

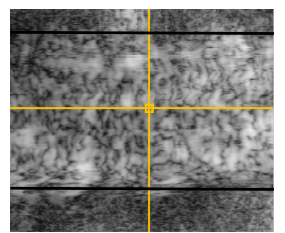

(b)

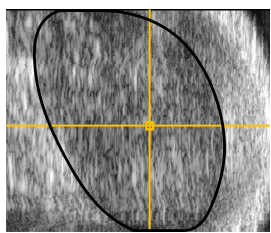

(c)
Fig. 1. Example of in-vivo 3D Achilles tendon acquired with the Vevo 2100 and the 3D acquisition module. The acquisition is always performed along the sagittal plane (a) which is aligned with the major loading direction of the tendon; (bc) correspond to concatenation planes, coronal and transverse respectively. Solid line delimits the tendon and dashed line represent tendon fibers.

Newton L-BFGS was used as optimization scheme and the used transformation model was affine. Taking into account the local similarity of speckle in US images and the limited capture range for image registration approaches, frames with large strain values cannot be registered directly to undeformed frames. Therefore we opted for a pair-wise image registration strategy as represented at the bottom axis of Fig. 2. Once this is completed, each pair-wise transformation is used to compose the deformation between the deformed and the undeformed images, as represented at the top axis of Fig. 2. The above presented registration method was implemented using elastix [3,4].

\subsection{Validation Data}

A recurring challenge in the literature on US strain quantification is the difficulty to establish a reliable ground-truth [5]. In this work we therefore generated two types of ground-truth data with associated deformation mechanisms to validate the proposed strain estimation method:

Computer-simulated data A 3D anatomical model of a tendon was constructed by approximating the tendon components (Fig.1(a)) to point clouds of cylindrical shapes. Lowest intensity values were allocated to surrounding tissue points. Medium and high intensity values were allocated to tendon unit and tendon fiber points, respectively. Scatter points mimicking contact gel particles were also added around the constructed model.

A linear strain deformation was then applied to the model, displacing scatterers in the strain direction and towards the center of the volume. Gel scatterers were displaced randomly. The applied longitudinal strain was simulated within a physiologic realistic range of $0 \%$ to $2 \%$ strain, along the lateral direction, with incremental intervals of $0.25 \%$ and the model was defined as an elastic and incompressible material.

Strain was estimated using eq. 1. $\varepsilon_{y}, \varepsilon_{x}$ and $\varepsilon_{z}$ correspond with the strain along the lateral, axial and elevation direction of the US transducer, respectively.

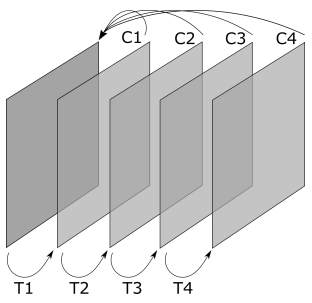

Fig. 2. Image registration strategy. The bottom axis $\left(T_{n}\right)$ represents the pair-wise image registration and the top axis $\left(C_{n}\right)$ represents the composition process to the first frame.

$$
\begin{aligned}
& \varepsilon_{y}=\frac{L_{1}}{L_{0}}-1 * 100 \\
& \varepsilon_{x}=\varepsilon_{z}=\frac{R_{1}}{R_{0}}-1 * 100, \quad R_{1}=R_{0} \sqrt{\frac{L_{0}}{L_{1}}}
\end{aligned}
$$

$L 0 / R 0$ and $L 1 / R 1$ correspond with the initial and final height/ radius of the phantom along the axial and elevation directions.

The deformed model was used as input to a US simulation platform called Field II $[6,7]$ with US parameters optimally mimicking the Vevo2100 US scanner used in our framework.

Physical phantom data Six agar-based phantoms with amorphous graphite scattering were constructed to validate the proposed framework. The used cylindrical mold with dimensions of $7 \mathrm{~cm} \times 7 \mathrm{~cm}$. Phantoms were compressed by means of a mechanical device up to a maximum of $4.28 \%$ strain (0,5\% increments) along the lateral direction. A increment different than the one used in the previous experiment was used due to the lower precision of the mechanical device. Also in this situation, strain was estimated using eq. 1.

\subsection{Analysis}

Due to the novelty of the framework, we first analysed its accuracy followed by a validation of the 3D strain calculations. 2D strain, using the same simulated data, was also validated.

Accuracy analysis of acquisition This acquisition systems accuracy was evaluated by repeatedly scanning an agarphantom (5 times) while using different contact media. Care was taken that the acquisition parameters were unchanged throughout the repetitions. The contact media used to do so were: water, gel, gel-pad and for the last test the transducer applied pressure to the gel pad. The images collected in each test condition were registered pair-wise among each-other using the presented image registration method. Deviations from $0 \%$ strain estimations were considered as inaccuracy errors.

Validation of 3D strain estimation Next we validated the performance of the presented 3D image registration method for strain calculation for both types of data and along each direction. Strain calculations are extracted from 
Table 1. Statistical analysis of results obtained for for phantom and simulated data. Correlation coefficients (CC) and regression slope (RS)) and Bland-Altman bias (BA-bias) and Limits-of-Agreement (LOA).

\begin{tabular}{|c|c|c|c|c|c|c|c|c|c|}
\hline & & \multicolumn{4}{|c|}{ Simulation } & \multicolumn{4}{|c|}{ Phantom } \\
\hline & & $\mathrm{CC}$ & RS & BA-bias(\%) & BA- LOA(\%) & $\mathrm{CC}$ & RS & BA-bias(\%) & BA- LOA(\%) \\
\hline \multirow{3}{*}{$3 \mathrm{D}$} & $\varepsilon_{x}$ & 1 & 1.08 & 0.03 & $\begin{array}{llll}-0.02 & \ldots .08 & 0.0\end{array}$ & 0.99 & 0.75 & -0.04 & $-0.39 . .0 .30$ \\
\hline & $\varepsilon_{y}$ & 1 & 1.02 & -0.03 & $-0.05 \ldots-0.01$ & 0.99 & 0.84 & 0.23 & $-0.23 \ldots 0.69$ \\
\hline & $\varepsilon_{z}$ & 0.99 & 1.91 & 0.43 & $\begin{array}{lll}-0.12 \ldots & 0.98\end{array}$ & 0.96 & 1.92 & 1.06 & $-1.14 \ldots 3.25$ \\
\hline \multirow{2}{*}{$2 \mathrm{D}$} & $\varepsilon_{x}$ & 1 & 1.32 & 0.22 & $0.02 \ldots 0.42$ & - & - & - & - \\
\hline & $\varepsilon_{y}$ & 1 & 1.19 & -0.30 & $-0.54 \ldots-0.05$ & - & - & - & - \\
\hline
\end{tabular}

the principal diagonal elements of the resulting affine matrix generated by each registration process. Ground-truth strain along the lateral direction was directly obtained from the deformation mechanism. Ground-truth strain along the axial and elevation direction were obtained using eq. 1. Strain estimations were compared against the ground-truth using correlation coefficients (CC), regression slope (RS) and Bland-Altman analysis, bias (BA-bias) and Limits-ofAgreement (LOA). Root mean squared error (RMSE) and normalized squared error (NSE) were computed to allow comparison against results reported in the literature.

Validation of 2D strain estimation For 2D strain estimation, the central slice of every 3D simulated volume was selected. These images were then registered using the same registration methodology presented before. 2D strain estimation for phantom data was not possible due to motion artefacts introduced by the semi-dynamic acquisition. Due to these artefacts, the central slice of one of the volumes not always corresponds to the central slice of the others. RMSE and NSE along axial and lateral direction were also computed.

\section{RESULTS}

Accuracy analysis of acquisition For the water and gel test conditions, results show equivalent errors (ranging between $-0.15 \%$ and $0.1 \%$ strain) along the three directions. The test using the gel-pad however shows an increment of the error along the elevation direction. The largest errors are obtained when pressure is applied, ranging between $-3.25 \%$ and $3.25 \%$ of strain.

Validation of 3D strain estimation Validation based on simulated data resulted in the lowest Bland-Altman bias, Bland-Altman limits-of-agreement and the highest correlation coefficient. Strain calculations along the lateral direction typically resulted in the lowest errors, whereas the highest errors were found along the elevation direction. Tables 1 summarize these results. Root mean squared error and normalized squared error for this registration are presented in section 3D of table 2.

Validation of 2D strain estimation 2D strain calculations were also evaluated using correlation and Bland-Altman deviations as shown in table 1 , section $2 \mathrm{D}$. The obtained root mean squared error and normalized squared error for simulation data are presented in section $2 \mathrm{D}$ of table 2 .

\section{DISCUSSION}

Our accuracy analysis showed that errors obtained while using water or gel as contact media are negligible. The same does not happen for the gel-pad and pressure test conditions. In these, errors along the elevation direction have a considerable increment which may be caused by shadows or by the increased friction on the scanned surface. Friction is responsible for the dragging of the motor or this to skip some steps, producing skewed images or deforming the phantom.

When considering 3D strain estimation, the lowest errors were found along the lateral direction. This was expected as this is the major deformation mechanism. Furthermore, strain along this direction is directly controlled by the deformation direction. For the axial and elevation direction (for phantom data) the ground-truth is derived from the compression applied along the lateral direction. This assumption would be correct if the phantom had a Poisson's ratio of $\approx 0.5$. If this is not the case, the quality of ground-truth data may be negatively affected. Nevertheless, we believe the obtained accuracy in the compression/expansion direction to be indicative of a good registration performance assisting in the good estimation of strain along the lateral direction.

When evaluating the different types of data, simulation data shows the highest correlation coefficients, regression slope and lowest Bland-Altman bias values as well as lowest RMSE and NSE. These results are to be expected because this type of data is noise free and has a linear imposed deformation.

At last, the combination of the 3D acquisition with the 3D image registration method suggests a reduction of the out-ofplane effect, as shown in table 1 , where an increment of the Bland-Altman bias and LOA errors were obtained for the 2D strain estimations. This is also represented in table 2 by the lowest RMSE obtained for 3D simulated data.

For best knowledge of the authors there is not literature reporting 3D tendon strain estimation or 2D strain estimations on simulated data. Due to that, comparison is only possible using the reported results for $2 \mathrm{D}$ phantom strain estimations. 
Table 2. Root mean squared error (RMSE) and normalized squared error (NSE) for phantom and simulated data.

\begin{tabular}{|c|c|c|c|c|c|}
\hline & \multicolumn{2}{|c|}{ Simulation } & \multicolumn{2}{|c|}{ Phantom } \\
\hline & & RMSE & NSE & RMSE & NSE \\
\hline \multirow{3}{*}{$3 \mathrm{D}$} & $\varepsilon_{x}$ & 0.02 & $-9.30 e^{-5}$ & 0.76 & $5.86 e^{-4}$ \\
\hline & $\varepsilon_{y}$ & 0.05 & $1.03 e^{-5}$ & 0.99 & $-8.07 e^{-4}$ \\
\hline & $\varepsilon_{z}$ & 0.31 & -0.04 & 1.02 & $3.60 e^{-3}$ \\
\hline \multirow{2}{*}{$2 \mathrm{D}$} & $\varepsilon_{x}$ & 0.04 & -0.09 & - & - \\
\hline & $\varepsilon_{y}$ & 0.42 & $7.90 e^{-4}$ & - & - \\
\hline
\end{tabular}

The RMSE, using simulated data along the lateral direction $(R M S E=0.05)$, obtained using our method shows better accuracy than the one reported by Slane et al.[5] using phantom data, both for their own method $(R M S E=$ $0.25)$ as well as for a method using digital image correlation (DIC) $(R M S E=0.53)$. Brown et al. [8] reported a NSE, using phantom data, for their developed method $\left(N S E=1.22 e^{-3}\right)$ as well as for a so called Multiscale method $\left(N S E=2.41 e^{-3}\right)$. Also in this comparison, 3D strain estimations using simulation data performed better. Considering phantom data, our method $\left(N S E=-8.07 e^{-4}\right.$ presented better results than the one of Brown et. al. Nevertheless, for this type of data, our method performed less well than Slane et al. These results could be explained by differences in the experimental setup. Firstly, our phantom was constructed using agar and had graphite powder $(6-20 \mu \mathrm{m}$ of diameter) functioning as scatterer method. On the other hand, Slane et al. constructed their phantom using Polyvinyl chloride-plastisol with randomly dispersed glass beads (30$50 \mu \mathrm{m}$ of diameter). Moreover, the deformation mechanism used was different i.e. compression/stretching.

\section{CONCLUSION}

Strain estimation on tendons has been a hot topic in the musculoskeletal research for a long time however there is still no available solution for accurate intratendinous (local) strain estimation.

The method presented herein steps away from conventional low-frequency 2D US system to a high-frequency 3D US system. In addition, a global image registration method was developed and optimized. From a methodological point of view, 3D US acquisition using the Vevo2100 system is challenging requiring close attention to acquisition conditions. Simulation results showed better accuracy for strain estimations using 3D data than $2 \mathrm{D}$ data revealing the impact of the out-of-plane effect. When compared to the state-ofthe-art, our method performed as accurate as most but not all, remaining however challenges.

Further tests should investigate the differences found between 3D and 2D strain estimations and the impact of out-ofplane effect. In addition, 3D strain estimation tests should be performed on ex-vivo biological tissue. A similar accuracy is expected since the image acquisition setup would be the same and the ex-vivo tendon is expected to have a global uniform deformation. This would then prove the robustness of this method to different types of data allowing to move towards the estimation of in-vivo intratendinous strain.

Furthermore, simulation of local strain deformation, following a biological representative model, would be of great contribution for validation of initial local strain estimations.

\section{ACKNOWLEDGEMENTS}

This work was supported by KU Leuven's Concerted Research Action GOA/11/006

\section{REFERENCES}

[1] S. P. Arnoczky, M. Lavagnino, and M. Egerbacher, "The mechanobiological aetiopathogenesis of tendinopathy: is it the over-stimulation or the under-stimulation of tendon cells?," Int. J. Exp. Pathol., vol. 88, no. 4, pp. 217-226, aug 2007 .

[2] A. Arndt, A. Bengtsson, M. Peolsson, A. Thorstensson, and T. Movin, "Non-uniform displacement within the Achilles tendon during passive ankle joint motion.," Knee Surg. Sports Traumatol. Arthrosc., vol. 20, no. 9, pp. 1868-74, oct 2012.

[3] S. Klein, M. Staring, K. Murphy, M. A. Viergever, and J. P. Pluim, "elastix: a toolbox for intensity-based medical image registration.," IEEE Trans. Med. Imaging, vol. 29, no. 1, pp. 196-205, jan 2010.

[4] D. P. Shamonin, E. E. Bron, B. P. Lelieveldt, M. Smits, S. Klein, and M. Staring, "Fast parallel image registration on CPU and GPU for diagnostic classification of Alzheimer's disease.," Front. Neuroinform., vol. 7, pp. 50, jan 2013.

[5] L. Chernak Slane and D. G. Thelen, "The use of 2D ultrasound elastography for measuring tendon motion and strain.," J. Biomech., vol. 47, no. 3, pp. 750-4, feb 2014.

[6] J. A. Jensen and N. B. Svendsen, "Calculation of pressure fields from arbitrarily shaped, apodized, and excited ultrasound transducers.," IEEE Trans. Ultrason. Ferroelectr. Freq. Control, vol. 39, no. 2, pp. 262-7, jan 1992.

[7] J. A. Jensen, "FIELD: A Program for Simulating Ultrasound Systems," 1996.

[8] P. G. Brown, J. Alsousou, A. Cooper, M. S. Thompson, and J. A. Noble, "The AutoQual ultrasound elastography method for quantitative assessment of lateral strain in post-rupture Achilles tendons.," J. Biomech., vol. 46, no. 15, pp. 2695-700, oct 2013. 